Annals of Pure and Applied Mathematics

Vol. 18, No. 1, 2018, 27-35

ISSN: 2279-087X (P), 2279-0888(online)

Published on 17 July 2018

Annals of

www.researchmathsci.org

DOI: http://dx.doi.org/10.22457/apam.v18n1a4

Pure and Applied

Mathematics

\title{
A Type of the Cauchy-Euler Equations: A Unique Real Root
}

\section{Wichai Jisabuy and Gumpon Sritanratana ${ }^{1}$}

Department of Mathematics, Rajabhat Mahasarakham University

Mahasarakham 44000, Thailand. E-mail: wichai.jisabuy@gmail.com

${ }^{1}$ Corresponding author. E-mail: sgumpon@ gmail.com

Received 2 June 2018; accepted 14 July 2018

Abstract. In this research, we give the family of all homogeneous Cauchy-Euler equations such that each equation has general solution depending only on a unique real number.

Keywords: Linear Differential Equations, Cauchy-Euler equations.

AMS Mathematics Subject Classification (2010): 34A30, 34B30

\section{Introduction}

Consider a homogeneous Cauchy-Euler equation of order $n$ of the form

$$
a_{n} x^{n} \frac{d^{n} y}{d x^{n}}+a_{n-1} x^{n-1} \frac{d^{n-1} y}{d y^{n-1}}+\ldots+a_{1} x \frac{d y}{d x}+a_{0} y=0 .
$$

where $a_{0}, a_{1}, \ldots, a_{n}$ are real numbers with $a_{n} \neq 0$. Details for methods to find solutions of the equation (1.1) was explained in [2, 4, 5, 8]. Moreover, Sabuwala and Leon [6] studied the particular solution for the most general $n$-th order Euler differential equation when the non-homogeneity is a polynomial. They found a formula which can be used to compute the unknown coefficients in the form of the particular solution. It is well known that the general solution of (1.1) can be found from the characteristic equation

$$
\sum_{j=1}^{n} a_{j} \prod_{i=1}^{j}(m-i+1)+a_{0}=0
$$

of the linear ordinary differential equation with constant coefficients

$$
\left(\sum_{j=1}^{n} a_{j} \prod_{i=1}^{j}\left(\frac{d}{d t}-i+1\right)+a_{0}\right) y=0,
$$

where $t=\ln x$. In general, the general solution of any homogeneous Cauchy-Euler equations depends on zeros of the polynomial $\sum_{j=1}^{n} a_{j} \prod_{i=1}^{j}(m-i+1)+a_{0}$. 
Wichai Jisabuy and Gumpon Sritanratana

The aim of this paper is to give the family of all Cauchy-Euler equations (1.1) such that $y=x^{\alpha} \sum_{i=1}^{n} c_{i} \ln ^{i-1} x$ is the general solution of $(1.1)$ on $(0, \infty)$ for some real number $\alpha$.

\section{Preliminary}

In this section, we shall give the related basic notions that can be found in $[1,5,7]$.

Let $n \in \mathbb{N}, a_{0}, a_{1}, \ldots, a_{n} \in \mathbb{R}$ with $a_{n} \neq 0$. An ordinary differential equation of the form

$$
a_{n} \frac{d^{n} y}{d x^{n}}+a_{n-1} \frac{d^{n-1} y}{d y^{n-1}}+\ldots+a_{1} \frac{d y}{d x}+a_{0} y=0
$$

is said to be a homogeneous linear ordinary differential equation with constant coefficients. By a transformation $y=e^{m x}$, where $m$ is a suitable number, the equation (2.1) is transformed into the polynomial equation

$$
a_{n} m^{n}+a_{n-1} m^{n-1}+\ldots+a_{1} m+a_{0}=0,
$$

which is said to be the characteristic equation of (2.1).

Theorem 2.1. [7] Let $n \in \mathbb{N}, a_{0}, a_{1}, \ldots, a_{n} \in \mathbb{R}$ with $a_{n} \neq 0$. Then the real numbers $\alpha$ is the zero of multiplicity $n$ of the polynomial $a_{n} m^{n}+a_{n-1} m^{n-1}+\cdots+a_{1} m+a_{0}=0$, if and on only if $y=x^{\alpha} \sum_{i=1}^{n} c_{i} \ln ^{i-1} x$ is the general solution of homogeneous linear ordinary differential equation (2.1) on $(0, \infty)$, where $c_{1}, \ldots, c_{n}$ are arbitrary constants.

A linear ordinary differential equation form

$$
a_{n} x^{n} \frac{d^{n} y}{d x^{n}}+a_{n-1} x^{n-1} \frac{d^{n-1} y}{d y^{n-1}}+\cdots+a_{1} x \frac{d y}{d x}+a_{0} y=0
$$

is called a homogeneous Cauchy-Euler equation.

The following theorem tells us that each Cauchy-Euler equation (2.2) can be transformed into linear ordinary differential equation with constant coefficients by the transformation $x=e^{t}$.

Theorem 2.2. [5] Let $n \in \mathbb{N}, a_{0}, a_{1}, \ldots, a_{n} \in \mathbb{R}$ with $a_{n} \neq 0$. Then the transformation $x=e^{t}$ transforms equation (2.2) into the equation

$$
\left(\sum_{j=1}^{n} a_{j} \prod_{i=1}^{j}\left(\frac{d}{d t}-i+1\right)+a_{0}\right) y=0,
$$

and the inverse transformation $t=\ln x$ transforms equation (2.4) into (2.2). 
A Type of the Cauchy-Euler Equation: A Unique Real Root

Corollary 2.1. [7] Let $n \in \mathbb{N}$ and $a_{0}, a_{1}, \ldots, a_{n} \in \mathbb{R}$ with $a_{n} \neq 0$. Then the transformation $x=e^{m t}$ transforms equation (2.4) into the equation

$$
\sum_{j=1}^{n} a_{j} \prod_{i=1}^{j}(m-i+1)+a_{0}=0 .
$$

Theorem 2.3. [5] Let $n \in \mathbb{N}, a_{0}, a_{1}, \ldots, a_{n} \in \mathbb{R}$ with $a_{n} \neq 0$. Then the real numbers $\alpha$ is the zero of multiplicity $n$ of the polynomial $\sum_{j=1}^{n} a_{j} \prod_{i=1}^{j}(m-i+1)+a_{0}$ if and only if $y=x^{\alpha} \sum_{i=1}^{n} c_{i} \ln ^{i-1} x$ is the general solution of Cauchy-Euler equation (2.2) on $(0, \infty)$, where $c_{1}, \ldots, c_{n}$ are arbitrary constants.

\section{Main theorems}

Definition 3.1. For each $j, k \in \mathbb{N}$ with $j \leq k$ we define

$$
\begin{gathered}
N_{k}:=\{1,2, \ldots, k\}, \\
P_{j, k}:=\left\{a_{1} a_{2} \cdots a_{j}: a_{1}, a_{2}, \ldots, a_{j} \in N_{k} \text { and } a_{1}<a_{2}<\cdots<a_{j}\right\}, \\
N_{j, k}:=\sum_{p \in P_{j, k}} p, \quad N_{0,0}:=1 \text { and } N_{0, k}:=1,
\end{gathered}
$$

and for every integers $j, k$ with $k>j$ we define $N_{k, j}:=0$.

For example; $N_{2,3}=1 \cdot 2+1 \cdot 3+2 \cdot 3=11$.

Form above definition, it is important to note that

$$
k N_{k-1, k-1}=N_{k, k}
$$

for every positive integer $k$. In addition, we have the following applicable lemma.

Lemma 3.1. Let $n$ be a positive integer. Then

$$
N_{i, n}+(n+1) N_{i-1, n}=N_{i, n+1}
$$

for all $i=1,2,3, \ldots, n$.

Proof: Let all $i=1,2,3, \ldots, n$ and

$$
Q_{i, n+1}=\left\{a_{1} a_{2} \cdots a_{i-1} \cdot(n+1): a_{1}, a_{2}, \ldots, a_{i-1} \in N_{n}, a_{1}<\cdots<a_{i-1}\right\}
$$

Before we proof (3.1), We shall prove that $P_{i, n}$ and $Q_{i, n+1}$ form a partition of $P_{i, n+1}$, that is $P_{i, n} \cap Q_{i, n+1}$ is empty and $P_{i, n} \cup Q_{i, n+1}=P_{i, n+1}$. For $P_{i, n} \cap Q_{i, n+1}$ is empty we suppose, to the contrary, that $P_{i, n} \cap Q_{i, n+1}$ is not empty. We can let $b \in P_{i, n} \cap Q_{i, n+1}$. Since $b \in Q_{i, n}$, there exist $a_{1}, a_{2}, \ldots, a_{i-1} \in N_{n}$ such that $a_{1}<a_{2}<\cdots<a_{i-1}$ and 
Wichai Jisabuy and Gumpon Sritanratana

$b=a_{1} a_{2} \cdots a_{i-1} \cdot(n+1)$ but $b \in P_{i, n}$ it follows that $a_{1}, a_{2}, \ldots, a_{i-1}, n+1 \in N_{n}$ and therefore $n+1 \in N_{n}$ which is a contradiction.

Now we shall prove that $P_{i, n} \cup Q_{i, n+1}=P_{i, n+1}$. We see that $P_{i, n} \cup Q_{i, n+1} \subset P_{i, n+1}$. since both $P_{i, n}$ and $Q_{i, n+1}$ are subsets of $P_{i, n+1}$. Now we shall show that $P_{i, n+1} \subset P_{i, n} \cup Q_{i, n+1}$. Let $b \in P_{i, n+1}$. Then there exist $a_{1}, a_{2}, \ldots, a_{i} \in N_{n+1}$ such that $b=a_{1} a_{2} \cdots a_{i}$ and $a_{1}<a_{2}<\cdots<a_{i}$. Therefore $a_{i} \leq n$ or $a_{i}=n+1$ since $a_{i} \in N_{n+1}$.

Case 1. Let $a_{i} \leq n$. Then $a_{1}, a_{2}, \ldots, a_{i} \in N_{n}$ since $a_{1}<a_{2}<\cdots<a_{i}$. Therefore $b=a_{1} a_{2} \cdots a_{i} \in P_{i, n}$ and thus $b \in P_{i, n} \cup Q_{i, n+1}$. Consequently, $P_{i, n+1} \subset P_{i, n} \cup Q_{i, n+1}$.

Case 2. Let $a_{i}=n+1$. Then $b=a_{1} a_{2} \cdots a_{i} \in Q_{i, n+1}$ since $a_{1}, a_{2}, \ldots, a_{i-1} \in N_{n}$. Therefore $b \in P_{i, n} \cup Q_{i, n+1}$. Consequently, $P_{i, n+1} \subset P_{i, n} \cup Q_{i, n+1}$.

Now $P_{i, n}$ and $Q_{i, n+1}$ form a partition of $P_{i, n}+1$.

Next, we consider

$$
N_{i, n}+(n+1) N_{i-1, n}=\sum_{p \in P_{i, n}} p+(n+1) \sum_{p \in P_{i-1, n}} p=\sum_{p \in P_{1, n}} p+\sum_{p \in Q_{i, n+1}} p .
$$

Because $P_{i, n}$ and $Q_{i, n+1}$, form a partition of $P_{i, n+1}$, we obtain

$$
\sum_{p \in P_{i, n}} p+\sum_{p \in Q_{i, n+1}}=\sum_{p \in P_{i, n} \cup Q_{i, n+1}} p=\sum_{p \in P_{i, n+1}} p=N_{i, n+1} .
$$

It follows that $N_{i, n}+(n+1) N_{i-1, n}=N_{i, n+1}$.

Lemma 3.2. Let $m \in \mathbb{C}$. For every $n \in \mathbb{N}$,

$$
\prod_{i=1}^{n}(m-i+1)=\sum_{i=0}^{n-1}(-1)^{i} N_{i, n-1} m^{n-i}
$$

Proof: We shall proof by mathematical induction on $n$. Since

$$
\prod_{i=1}^{1}(m-i+1)=m=(-1)^{0} N_{0,1-1} m^{1-0}=\sum_{i=0}^{1-1}(-1)^{i} N_{i, 1-1} m^{1-i},
$$

we obtain (3.2) is true for $n=1$.

Let $k \in \mathbb{N}$ be arbitrary. Suppose that

$$
\prod_{i=1}^{k}(m-i+1)=\sum_{i=0}^{k-1}(-1)^{i} N_{i, k-1} m^{k-i}
$$

is true. Since $\prod_{i=1}^{k+1}(m-i+1)=(m-k) \prod_{i=1}^{k}(m-i+1)$, by the inductive hypothesis we have

$$
\prod_{i=1}^{k+1}(m-i+1)=(m-k) \sum_{i=0}^{k-1}(-1)^{i} N_{i, k-1} m^{k-i}
$$


A Type of the Cauchy-Euler Equation: A Unique Real Root

$$
\begin{aligned}
= & \sum_{i=0}^{k-1}(-1)^{i} N_{i, k-1} m^{k-i+1}+\sum_{i=0}^{k-1}(-1)^{i+1} k N_{i, k-1} m^{k-i} \\
= & \sum_{i=0}^{k-1}(-1)^{i} N_{i, k-1} m^{k-i+1}+\sum_{j=1}^{k}(-1)^{j} k N_{j-1, k-1} m^{k-j+1} \\
= & \sum_{i=0}^{k-1}(-1)^{i} N_{i, k-1} m^{k-i+1}+\sum_{i=1}^{k}(-1)^{i} k N_{i-1, k-1} m^{k-i+1} \\
= & (-1)^{0} N_{0, k-1} m^{k+1}+\sum_{i=1}^{k-1}(-1)^{i} N_{i, k-1} m^{k-i+1}+\sum_{i=1}^{k-1}(-1)^{i} k N_{i-1, k-1} m^{k-i+1} \\
& +(-1)^{k} k[(k-1) !] m \\
= & (-1)^{0} N_{0, k-1} m^{k+1}+\sum_{i=1}^{k-1}(-1)^{i}\left(N_{i, k-1}+k N_{i-1, k-1}\right) m^{k-i+1}+(-1)^{k} k ! m .
\end{aligned}
$$

Therefore, by Lemma 3.1 and $k !=N_{k, k}$, we obtain

$$
\begin{aligned}
\prod_{i=1}^{k+1}(m-i+1) & =(-1)^{0} N_{0, k-1} m^{k+1}+\sum_{i=1}^{k-1}(-1)^{i} N_{i, k} m^{k-i+1}+(-1)^{k} N_{k, k} m \\
& =\sum_{i=0}^{k}(-1)^{i} N_{i, k} m^{k-i+1} .
\end{aligned}
$$

Thus (3.2) is true for $n=k+1$. The proof is complete by mathematical induction

Lemma 3.3 Let $m \in \mathbb{C}$ and $a_{1}, \ldots, a_{n} \in \mathbb{R}$ with $a_{n} \neq 0$. Then

$$
\sum_{j=1}^{n} a_{j} \prod_{i=1}^{j}(m-i+1)=a_{n} m^{n}+\sum_{j=1}^{n-1} m^{n-j} \sum_{i=0}^{j}(-1)^{i} N_{i, n+i-j-1} a_{n+i-j},
$$

for every $n \in \mathbb{N}$ with $n \geq 2$.

Prove: We prove by induction. For $n=2$, consider

$$
\begin{aligned}
\sum_{j=1}^{2} a_{j} \prod_{i=1}^{j}(m-i+1) & =a_{1} \prod_{i=1}^{1}(m-i+1)+a_{2} \prod_{i=1}^{2}(m-i+1) \\
& =a_{1} m+a_{2} m(m-1) \\
& =a_{2} m^{2}+\left(a_{1}-a_{2}\right) m \\
& =a_{2} m^{2}+\left((-1)^{0} N_{0,0} a_{1}+(-1)^{1} N_{1,1} a_{2}\right) m \\
& =a_{2} m^{2}+m \sum_{i=0}^{1}(-1)^{i} N_{i, i} a_{1+i} \\
& =a_{2} m^{2}+\sum_{j=1}^{1} m^{2-j} \sum_{i=0}^{j}(-1)^{i} N_{i, i-j+1} a_{2+i-j} .
\end{aligned}
$$

It follows that (3.3) is true for $n=2$.

Let $k \in \mathbb{N}$ with $k \geq 2$ and suppose that (3.2) is true for $n=k$, that is 
Wichai Jisabuy and Gumpon Sritanratana

$$
\sum_{j=1}^{k} a_{j} \prod_{i=1}^{j}(m-i+1)=a_{k} m^{k}+\sum_{j=1}^{k-1} m^{k-j} \sum_{i=0}^{j}(-1)^{i} N_{i, k+i-j-1} a_{k+i-j} .
$$

We must prove that (3.2) is true for $n=k+1$. Consider

$$
\sum_{j=1}^{k+1} a_{j} \prod_{i=1}^{j}(m-i+1)=\sum_{j=1}^{k} a_{j} \prod_{i=1}^{j}(m-i+1)+a_{k+1} \prod_{i=1}^{k+1}(m-i+1) .
$$

Therefore, by inductive hypothesis and Lemma 3.2, we obtain

$$
\begin{aligned}
& \sum_{j=1}^{k+1} a_{j} \prod_{i=1}^{j}(m-i+1)=a_{k} m^{k}+\sum_{j=1}^{k-1} m^{k-j} \sum_{i=0}^{j}(-1)^{i} N_{i, k+i-j-1} a_{k+i-j}+a_{k+1} \sum_{i=0}^{k}(-1)^{i} N_{i, k} m^{k-i+1} \\
& =a_{k} m^{k}+\sum_{j=1}^{k-1} m^{k-j} \sum_{i=0}^{j}(-1)^{i} N_{i, k+i-j-1} a_{k+i-j}+\sum_{i=0}^{k}(-1)^{i} N_{i, k} a_{k+1} m^{k-i+1} \\
& =a_{k} m^{k}+\sum_{j=1}^{k-1} m^{k-j} \sum_{i=0}^{j}(-1)^{i} N_{i, k+i-j-1} a_{k+i-j}+\left[a_{k+1} m^{k+1}+(-1)^{1} N_{1, k} a_{k+1} m^{k}\right. \\
& \left.+(-1)^{2} N_{2, k} a_{k+1} m^{k-1}+\cdots+(-1)^{k-1} N_{k-1, k} a_{k+1} m^{2}+(-1)^{k} N_{k, k} a_{k+1} m\right] \\
& =a_{k+1} m^{k+1}+(-1)^{0} N_{0, k-1} a_{k} m^{k}+\sum_{i=0}^{1}(-1)^{i} N_{i, k+i-2} a_{k+i-1} m^{k-1} \\
& +\sum_{i=0}^{2}(-1)^{i} N_{i, k+i-3} a_{k+i-2} m^{k-2}+\cdots+\sum_{i=0}^{k-1}(-1)^{i} N_{i, i} a_{i+1} m \\
& +(-1)^{1} N_{1, k} a_{k+1} m^{k}+(-1)^{2} N_{2, k} a_{k+1} m^{k-1}+\cdots+(-1)^{k-1} N_{k-1, k} a_{k+1} m^{2} \\
& +(-1)^{k} N_{k, k} a_{k+1} m \\
& =a_{k+1} m^{k+1}+m^{k}\left[(-1)^{0} N_{0, k-1} a_{k}+(-1)^{1} N_{1, k} a_{k+1}\right] \\
& +m^{k-1}\left[\sum_{i=0}^{1}(-1)^{i} N_{i, k+i-2} a_{k+i-1}+(-1)^{2} N_{2, k} a_{k+1}\right] \\
& +\cdots+m\left[\sum_{i=0}^{k-1}(-1)^{i} N_{i, i} a_{i+1}+(-1)^{k} N_{k, k} a_{k+1}\right] \\
& =a_{k+1} m^{k+1}+m^{k} \sum_{i=0}^{k-1}(-1)^{i} N_{i, k+i-1} a_{k+i}+m^{k-1} \sum_{i=0}^{2}(-1)^{i} N_{i, k+i-2} a_{k+i-1} \\
& +\cdots+m^{2} \sum_{i=0}^{k-1}(-1)^{i} N_{i, i+1} a_{i+2}+m \sum_{i=0}^{k}(-1)^{i} N_{i, i} a_{i+1} \\
& =a_{k+1} m^{k+1}+\sum_{j=1}^{k} m^{k+1-j} \sum_{i=0}^{j}(-1)^{i} N_{i, k+i-j} a_{k+i-j+1} .
\end{aligned}
$$

Thus (3.3) is true for $n=k+1$. The proof is complete.

From the above lemma, adding both sides of the equation (3.3) by $a_{0}$, we obtain 
A Type of the Cauchy-Euler Equation: A Unique Real Root

$$
\sum_{j=1}^{n} a_{j} \prod_{i=1}^{j}(m-i+1)+a_{0}=a_{n} m^{n}+\sum_{j=1}^{n-1} m^{n-j} \sum_{i=0}^{j}(-1)^{i} N_{i, n+i-j-1} a_{n+i-j}+a_{0},
$$

and thus by Theorem 2.3, we obtain the following corollary.

Corollary 3.1. Let $n \in \mathbb{N}$ and $m, \alpha, a_{0}, a_{1}, \ldots, a_{n} \in \mathbb{R}$ with $a_{n} \neq 0$. Then $\alpha$ is the zero of multiplicity $n$ of the polynomial

$$
a_{n} m^{n}+\sum_{j=1}^{n-1} m^{n-j} \sum_{i=0}^{j}(-1)^{i} N_{i, n+i-j-1} a_{n+i-j}+a_{0}
$$

if on only if $y=x^{\alpha} \sum_{i=1}^{n} c_{i} \ln ^{i-1} x$ is the general solution of the Cauchy-Euler equation

$$
a_{n} x^{n} \frac{d^{n} y}{d x^{n}}+a_{n-1} x^{n-1} \frac{d^{n-1} y}{d y^{n-1}}+\cdots+a_{1} x \frac{d y}{d x}+a_{0} y=0
$$

on the open interval $(0, \infty)$, where $c_{1}, \ldots, c_{n}$ are arbitrary constants.

Lemma 3.4. Let $n \in \mathbb{N}$ and $m, \alpha, a_{0}, a_{1}, \ldots, a_{n} \in \mathbb{R}$ with $a_{n} \neq 0$. Then

$$
a_{n} m^{n}+\sum_{j=1}^{n-1} m^{n-j} \sum_{i=0}^{j}(-1)^{i} N_{i, n+i-j-1} a_{n+i-j}+a_{0}=m^{n}+\sum_{j=1}^{n-1}(-1)^{j}\left(\begin{array}{c}
n \\
j
\end{array}\right) \alpha^{j} m^{n-j}+(-1)^{n} \alpha^{n}
$$

if and only if

$$
a_{0}=(-1)^{n} \alpha^{n}, a_{n}=1 \text { and } a_{k}=(-1)^{n-k}\left(\begin{array}{l}
n \\
k
\end{array}\right) \alpha^{n-k}+\sum_{i=0}^{n-k-1}(-1)^{i} N_{i+1, i+k} a_{i+k+1}
$$

for every $k=1,2, \ldots, n-1$.

Proof: Because the set of $1, m, m^{2}, \ldots, m^{n}$ are linearly independent on $\mathbb{R}$, we have (3.6) is true if and only if $a_{0}=(-1)^{n} \alpha^{n}, a_{n}=1$ and for every $k=1,2, \ldots, n-1$,

$$
\sum_{i=0}^{n-k}(-1)^{i} N_{i, i+k-1} a_{i+k}=(-1)^{n-k}\left(\begin{array}{c}
n \\
n-k
\end{array}\right) \alpha^{n-k}
$$

Since $N_{0, k-1}=1,\left(\begin{array}{c}n \\ n-k\end{array}\right)=\left(\begin{array}{l}n \\ k\end{array}\right)$ and

$$
\sum_{i=0}^{n-k}(-1)^{i} N_{i, i+k-1} a_{i+k}=N_{0, k-1} a_{k}+\sum_{i=1}^{n-k}(-1)^{i} N_{i, i+k-1} a_{i+k}=a_{k}+\sum_{i=1}^{n-k}(-1)^{i} N_{i, i+k-1} a_{i+k},
$$

from (3.8), we have

$$
a_{k}+\sum_{i=1}^{n-k}(-1)^{i} N_{i, i+k-1} a_{i+k}=(-1)^{n-k}\left(\begin{array}{l}
n \\
k
\end{array}\right) \alpha^{n-k} .
$$

Since $-\sum_{i=1}^{n-k}(-1)^{i} N_{i, i+k-1} a_{i+k}=\sum_{i=1}^{n-k}(-1)^{i+1} N_{i, i+k-1} a_{i+k}=\sum_{i=0}^{n-k-1}(-1)^{i} N_{i+1, i+k} a_{i+k+1}$, we obtain 
Wichai Jisabuy and Gumpon Sritanratana

$$
a_{k}=(-1)^{n-k}\left(\begin{array}{l}
n \\
k
\end{array}\right) \alpha^{n-k}+\sum_{i=0}^{n-k-1}(-1)^{i} N_{i+1, i+k} a_{i+k+1} .
$$

Therefore (3.8) and (3.9) are equivalent. This proof is complete.

By binomial theorem,

$$
(m-\alpha)^{n}=m^{n}+\sum_{j=1}^{n-1}(-1)^{j}\left(\begin{array}{l}
n \\
j
\end{array}\right) \alpha^{j} m^{n-j}+(-1)^{n} \alpha^{n},
$$

we obtain $\alpha$ is the zero of multiplicity $n$ of the polynomial

$$
m^{n}+\sum_{j=1}^{n-1}(-1)^{j}\left(\begin{array}{l}
n \\
j
\end{array}\right) \alpha^{j} m^{n-j}+(-1)^{n} \alpha^{n},
$$

the next corollary is a direct consequence of Lemma 3.4. and Corollary 3.1.

Corollary 3.2. Let $n \in \mathbb{N}$ and $m, \alpha, a_{0}, a_{1}, \ldots, a_{n} \in \mathbb{R}$ with $a_{n} \neq 0$. Then $\alpha$ is the zero of multiplicity $n$ of polynomial (3.4) if and only if (3.7) is true for every $k=1,2, \ldots, n-1$.

The next main theorem is an immediately consequence of Corollary 3.1 and Corollary 3.2.

Theorem 3.1. Let $n \in \mathbb{N}$ and $m, \alpha, a_{0}, a_{1}, \ldots, a_{n} \in \mathbb{R}$ with $a_{n} \neq 0$. Then $y=x^{\alpha} \sum_{i=1}^{n} c_{i} \ln ^{i-1} x$ is the general solution of the homogeneous Cauchy-Euler equation (3.5) on $(0, \infty)$, where $c_{1}, \ldots, c_{n}$ are arbitrary constants if and only if (3.7) is true for every $k=1,2, \ldots, n-1$.

The application of this theorem is to give a Cauchy-Euler equation of order $n$ from a given general solution on $(0, \infty)$ in the form $y=x^{\alpha} \sum_{i=1}^{n} c_{i} \ln ^{i-1} x$, where $\alpha$ is a given real number.

\section{Conclusion}

We give every Cauchy-Euler differential equation from its general solution that depends only on a given real numbers. In the future, we will devote our attention to the family of all Cauchy-Euler differential equations that have general solutions depending on several real numbers.

Acknowledgements. The authors are thankful to the reviewers for valuable comments and suggestions on the manuscript and thank the Faculty of Science and Technology, Rajabhat Mahasarakham University, Mahasarakham, Thailand for financial support. 
A Type of the Cauchy-Euler Equation: A Unique Real Root

\section{REFERENCES}

1. S.Ahmad and A.Ambrosetti, A Textbook on ordinary differential equations, Springer International Publishing Switzerland, (2015).

2. W.E.Boyce and R.C.DiPrima, Elementary differential equations and boundary value problems, Seventh edition, John Wiley \& Sons, Inc., New York-London-Sydney, (2001).

3. E.A.Coddington, An introduction to ordinary differential equations, Prentice-Hall Mathematics Series Prentice-Hall, Inc., Englewood Cliffs, N.J., (1961).

4. E.A.Coddington and N.Levinson, Theory of ordinary differential equations, McGraw-Hill Book Company, Inc., NewYork-Toronto-London, (1995).

5. S.W.Goode and S.A.Annin, Differential equations and linear algebra, fourth edition, Pearson Education, Inc., (2015).

6. A.H.Sabuwala and D.De Leon, Particular solution to the Euler-Cauchy equation with polynomial non-homogeneities, Discrete Contin. Dyn. Syst., Dynamical Systems, Differential Equations and Applications, 8th AIMS Conference. Suppl. Vol. II, (2011), 1271-1278.

7. D.A.Sanchez, Ordinary differential equations and stability theory an introduction, Dover Publications Inc., (1979).

8. D.Zill and W.Wright, Differential equations and boundary value problems, eighth edition, Brooks/Cole, Boston, (2013). 